\title{
Which Is a More Serious Factor to the Bandwidth of GI POF: Differential Mode Attenuation or Mode Coupling?
}

\author{
Takaaki Ishigure, Mariko Kano, and Yasuhiro Koike
}

\begin{abstract}
It is clarified for the first time that the effect of the mode coupling on the bandwidth characteristic is very small in the graded-index polymer optical fiber (GI POF) compared with in the step-index (SI) POF. Although there have been several trials regarding the theoretical prediction of bandwidth characteristics of the GI POF, a disagreement between measured and estimated properties was observed. The origin of disagreement has been generally explained as the mode coupling effect. However, it is clarified in this paper that the effect of the differential mode attenuation is the dominant factor of disagreement between measured and predicted bandwidth characteristics, while the effect of the mode coupling is small. In order to clarify the reason why the calculated bandwidth characteristics of the GI POF show a disagreement with that measured, differential mode delay and differential mode attenuation were investigated. It was found that the highest order mode in the poly methyl methacrylate (PMMA) base GI POF attenuated $17 \mathrm{~dB}$ compared with the lowest order mode through 100-m transmission. By taking into account this differential mode attenuation, an excellent agreement is observed between calculated and measured results of both the bandwidth characteristics and group delay, in which no mode coupling was considered.
\end{abstract}

Index Terms-Bandwidth, differential mode attenuation, graded-index polymer optical fiber (GI POF), modal dispersion, mode coupling.

\section{INTRODUCTION}

$\mathbf{T}$ RIGGERED by increasing demand accessing to Internet with high bit rate, much attention has been focused on the higher communication bandwidth even in the office and home network. Polymer optical fiber (POF) having much larger core than that of silica fiber has been expected to be the office and home-network media because the large core allows the use of inexpensive injection-molded plastic connector, which can dramatically decrease the total link cost. We proposed the high-bandwidth graded-index (GI) POF for the first time [1], and have reported its bandwidth characteristic [2]. Recently, with increasing interests on GI POF, there have been several reports concerning the dispersion properties of SI and GI POF's. The theoretical bandwidth properties of the POF's often have some amount of disagreement with those measured. The reason of the disagreement is generally explained by the mode cou-

Manuscript received January 20, 2000; revised April 14, 2000. This work is supported by the research fund of Plastic Optical Fiber Project from Telecommunications Advancement Organization (TAO) of Japan.

The authors are with the Faculty of Science and Technology, Keio University, Yokohama 223-8522, Japan. They are also with the Kanagawa Academy of Science and Technology, Yokohama 236-0004, Japan.

Publisher Item Identifier S 0733-8724(00)05763-7. pling. However, the detail modal property has not been analyzed.

In this paper, we clarify that the differential mode attenuation is the serious factor on the bandwidth characteristic of the POF. In the case of the conventional SI POF, we confirm that the mode coupling effect is very strong even in less than 50-m transmission. However, it is shown in this paper that the effect of mode coupling on the bandwidth characteristics of the GI POF is much smaller than that in the SI POF. We show a precise bandwidth prediction of the GI POF by considering the power distribution among the propagating modes and differential mode attenuation for the first time, resulting in a good agreement with the experimentally measured property.

\section{BANDWIDTH OF THE GI POF}

\section{A. Refractive Index Distribution Approximation}

It is well known that the modal dispersion of the multimode optical fiber can be minimized by the quadratic refractive index profile. We reported the interfacial-gel polymerization process could widely control the refractive index profile of the GI POF [2]. Fig. 1 shows the experimentally measured refractive index profile of the GI POF along with approximated profile. In order to investigate the relation between the refractive index profile and bandwidth characteristics of the GI POF, the refractive index profile is approximated by the power-law equation as described by (1)

$$
\begin{aligned}
n(r) & =n_{1}\left[1-2 \Delta\left(\frac{r}{a}\right)^{g}\right]^{1 / 2} \quad 0 \leq r \leq a \\
& =n_{2} \quad r \geq a
\end{aligned}
$$

where $n_{1}$ and $n_{2}$ are the refractive indexes of the core center and cladding respectively, $a$ is the core radius, and $\Delta$ is the relative index difference defined as

$$
\Delta=\frac{n_{1}^{2}-n_{2}^{2}}{2 n_{1}^{2}}
$$

The parameter $g$ called as index exponent determines the refractive index profile. The index exponents of various kinds of GI POF's prepared in our laboratory were estimated by using a least-square technique to fit to (2). By approximation of power-law form, the analytical solution of Maxwell's wave equation can be obtained by means of the Wentzel-Kramers-Brillouin (WKB) method. Approximated curve by (1) is also shown in Fig. 1 having 2.7 of index exponent $g$. However, the power-law approximation is not necessarily 


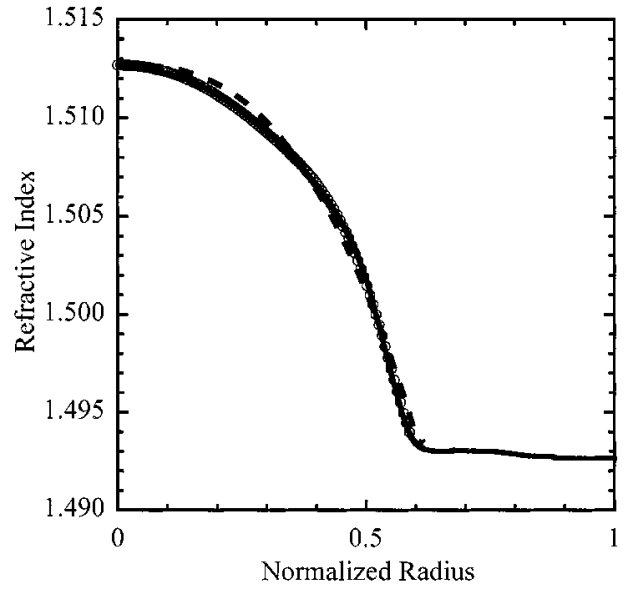

Fig. 1. Measured and approximated refractive index profiles of the PMMA base GI POF. - : Measured refractive index profile. - -: Approximated profile by power-law form when index exponent $g=2.7$. $\bigcirc$ : Approxiamted profile by polynomial form.

suitable for the index profile formed by the interfacial-gel polymerization technique. For instance, the measured index profile shown in Fig. 1 shows slight disagreement with the approximated curve by the power-law form when the index exponent $g=2.7$, although it was best fitted value by the least square method. If the index profile is deviated from the power-law curve, analytical solution of the Maxwell's equation can not be obtained, while numerical computation process enables the detail analysis of the bandwidth characteristic.

In order to accurately evaluate the dispersion characteristic of the GI POF whose index profile is deviated from power-law form, a numerical computation process based on WKB method was adopted [3], [4]. In the computation procedure, the index profile was described by ten terms polynomial function of the distance $r$ from the center axis of the fiber as shown in (3)

$$
\begin{array}{r}
n(r)=n_{1}\left[1-2 \cdot \Delta \cdot\left\{A_{10} \cdot\left(\frac{r}{a}\right)^{10}+A_{9} \cdot\left(\frac{r}{a}\right)^{9}\right.\right. \\
\left.\left.+\cdots+A_{2} \cdot\left(\frac{r}{a}\right)^{2}+A_{1} \cdot\left(\frac{r}{a}\right)\right\}\right]^{1 / 2}
\end{array}
$$

here $A_{10}, A_{9}, \ldots, A_{2}, A_{1}$ are constants independent of the wavelength of transmitting light.

\section{B. Dispersion Calculation}

In this numerical method, the group delay $\tau$ of the mode having the propagation constant $\beta$ can be expressed as

$$
\tau=\frac{L}{c} \frac{k}{\beta}\left\{\left[\int_{r_{1}}^{r_{2}} \frac{n^{2}+n k d n / d k}{R} d r\right] / \int_{r_{1}}^{r_{2}} \frac{d r}{R}\right\}
$$

where $n, c$, and $L$ signify the refractive index profile determined by (3), light velocity in vacuum, and the fiber length, respectively, and $k$ and $R$ are described in (5) and (6)

$$
\begin{aligned}
k & =\frac{2 \pi}{\lambda} \\
R & =\sqrt{n(r)^{2} k^{2}-\beta^{2}-\frac{\nu^{2}}{r^{2}}} .
\end{aligned}
$$

In (6), $\nu$ is called the azimuthal mode number. In (4), the poles of $r_{1}$ and $r_{2}$ in the integrand are defined as the solutions of (7)

$$
n(r)^{2} k^{2}-\beta^{2}-\frac{\nu^{2}}{r^{2}}=0 .
$$

After calculating the group delay $\tau$ of each mode, the time range of the group delay between the fastest and slowest mode is divided into 30 to 40 of time slots. The impulse response function was constructed by counting the number of modes whose group delay is involved in each time slots. It should be emphasized that the impulse response function was calculated by assuming that each propagating mode was transmitted having equal power. Subsequently, the output waveform was calculated by the convolution of the waveform of the measured real input pulse and calculated impulse response function.

\section{Bandwidth Measurement}

The bandwidths of the SI and GI POF's were measured by the time domain measurement method, in which the bandwidth was estimated by measuring the output pulse waveform when a narrow pulse was inserted into the fiber. As the light source, InGaAsP laser diode at $0.655-\mu \mathrm{m}$ wavelength was adopted. Input pulse generated by the pulse generator was inserted into the POF, and the output pulse was measured by sampling head (Hamamatsu OOS-01), and recorded and analyzed by sampling oscilloscope. Here, the launch condition of the POF is a very important issue to investigate the bandwidth characteristic. Generally, in bandwidth measurement of the multimode silica fiber, it has been preferred that the steady state mode power distribution is achieved because the steady state mode power distribution is already achieved in the real field of the silica base multimode fiber links that has more than several kilometer. Therefore, in order to achieve the steady state mode power distribution, all modes are fully excited with using the mode scrambler. In this paper, short step-index (SI) POF (1-m length) was used as the mode exciter to achieve the uniform launch condition of all modes. Pulsed signal from the LD was directly launched into the 1-m SI POF butting to the tested GI POF sample on the V-groove. Since the power distribution of the output end of 1-m SI POF is uniform in its core region, and the numerical aperture of the SI POF (0.5) is sufficiently higher than that of the GI POF $(0.2-0.3), 1-\mathrm{m}$ SI POF is considered as an ideal mode exciter for uniform launch.

\section{RESUlTS AND DiscUSSION}

\section{A. Bandwidth Prediction}

Bandwidth characteristic of the GI POF whose index profile is shown in Fig. 1 was calculated by WKB numerical computation method. For comparison of different approximation method of the refractive index profile, both power-law and polynomial forms were adopted. Calculated output waveforms are shown in Fig. 2 compared with that experimentally measured. In the case of power-law approximation, a large disagreement is observed between the measured and estimated waveforms, while in the case of polynomial form, the leading part (left side edge) of the pulse waveform is precisely predicted by numerical calculation. However, calculated waveform in the case of polynomial fitting 


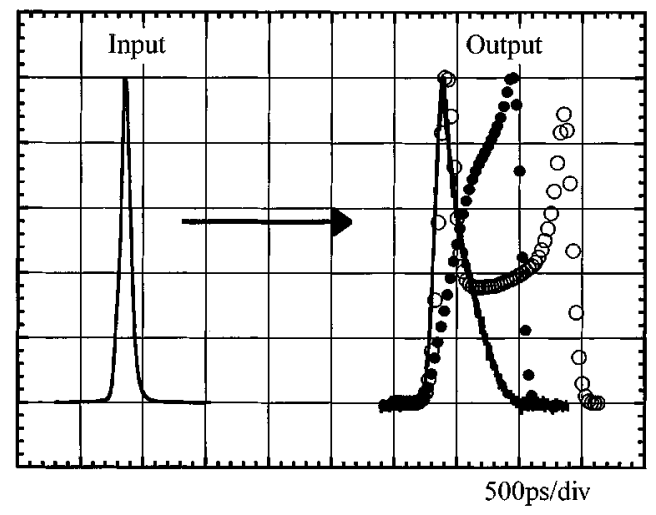

Fig. 2. Comparison of measured and calculated bandwidth characteristics of the GI POF whose index profile is shown in Fig. 1. Solid line: measured waveform. : Predicted waveform when index profile is approximated by the power-law form. $\bigcirc$ : Predicted waveform when index profile is approximated by polynomial form.

has two peaks, which differs from the measured one having only one peak.

In the case of the silica base GI multimode fiber, such a disagreement between measured and estimated bandwidth characteristics was observed. Furthermore, by recent several efforts to clarify the bandwidth characteristics of GI POF, it was reported that such a disagreement was attributed to the effect of the mode coupling. However, we clarified in this paper that the differential mode attenuation rather than the mode coupling strongly affects the bandwidth characteristic of the GI POF.

\section{B. Pulse Distortion with Respect to Fiber Length}

In the previous reports of silica base fiber, degree of the mode coupling was estimated by measuring the relation between the output pulse distortion and fiber length [5], [6]. It was already confirmed that no mode coupling provided the linear relation between the output pulse width $\sigma$ and fiber length $L$, while linear relation between $\sigma$ and $L^{\gamma}(0.5 \leq \gamma \leq 1)$ was obtained when a strong mode coupling existed. We investigated the relation between the output pulse width and the fiber length of the PMMA base GI POF. The result is shown in Fig. 3. Abscissa and ordinate of Fig. 3 are logarithms of fiber length $L$ and the r.m.s output pulse width $\sigma$ calculated by (8), respectively

$$
\sigma^{2}=\frac{\int_{-\infty}^{\infty}(t-\tau)^{2}(t) d t}{\int_{-\infty}^{\infty} t P(t) d t}
$$

where $P(t)$ is the waveform of the output pulse as a function of time $t$, and $\tau$ is defined as

$$
\tau=\frac{\int_{-\infty}^{\infty} t P(t) d t}{\int_{-\infty}^{\infty} P(t) d t} .
$$

As shown in Fig. 3, little pulse broadening was observed in less than 20-m fiber length. On the other hand, the slope of the relation between $\sigma$ and $L$ is 0.85 (less than 1) in the region where $L$ is longer than $20 \mathrm{~m} \mathrm{[5],} \mathrm{[6].} \mathrm{This} \mathrm{result} \mathrm{indicates} \mathrm{that} \mathrm{the} \mathrm{mode}$

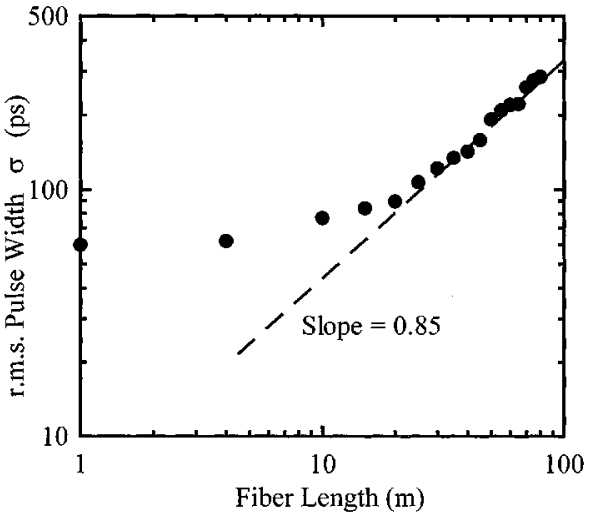

Fig. 3. Fiber length dependence of r.m.s. output pulse width from the GI POF. : experimentally measured data.

coupling seems to exist in the GI POF from 20 to 100-m length if we refer the previous works in the silica base multimode fiber. This issue would be clarified in the next article. Our question in this paper is if we can say the mode coupling exists in the GI POF only from Fig. 3. Our argument is that the differential mode attenuation may provide similar relation between $\sigma$ and $L$ as that when mode coupling exists. Therefore, we investigate the effect of the differential mode attenuation on the bandwidth characteristic. The relation between $\sigma$ and $L$ was investigated in many GI POF samples and it was found that exponent $\gamma$ varied from 0.7 to 0.9 . The origin of the variation in $\gamma$ value is much complicated. Both differential mode attenuation and mode coupling decreases the $\gamma$ value. Therefore, fiber length dependence of near-field pattern (NFP) and far-field pattern (FFP) data are required in addition to $\gamma$ value in order to accurately investigate the origin of lower $\gamma$ value than 1.0. We discuss these NFP and FFP issues in the following section.

\section{Differential Mode Delay}

The group delay of each mode in the GI POF was investigated by the measurement of differential mode delay (DMD). In the DMD measurement, optical pulse is coupled into the GI POF via a $1-\mathrm{m}$ single mode silica fiber in order to launch a specified mode group of the GI POF. By shifting the position of the single mode fiber butted to the GI POF from the core center to the periphery, each mode from the low order to high order can be independently launched. Schematic representation of experimental setup is shown in Fig. 4. The results of the DMD measurement in 100-m GI POF are shown by solid lines in Fig. 5. Here, the lengths $0,130, \ldots 190 \mu \mathrm{m}$ shown in Fig. 5 signify the distance (d) from the core center to launching position of the single mode fiber. Several GI POF samples with different refractive index profiles were adopted for DMD measurement. Since the length of tested fiber is limited to less than $100 \mathrm{~m}$ because of the large intrinsic attenuation, only a little time delay between the fastest and the slowest modes can be observed if the refractive index profile of the GI POF is optimum. Consequently, it becomes impossible to distinguish the DMD. Therefore, the GI POF whose refractive index profile is slightly deviated from the optimum profile was adopted in order to identify the group delay of each modes (group delay between the fastest and the slowest modes are obvious). The refractive index profile 


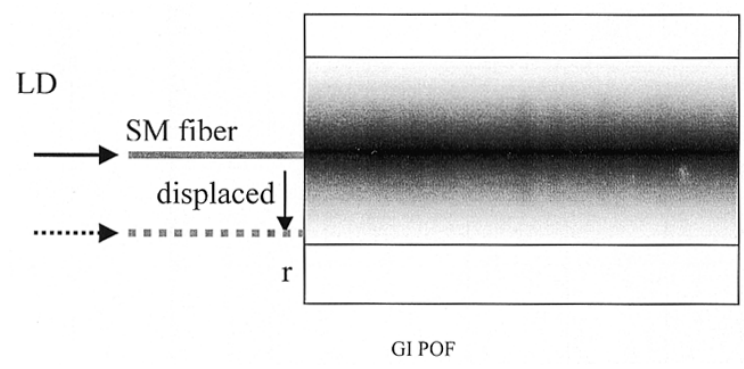

Fig. 4. Schematic representation of differential mode delay (DMD) measurement.

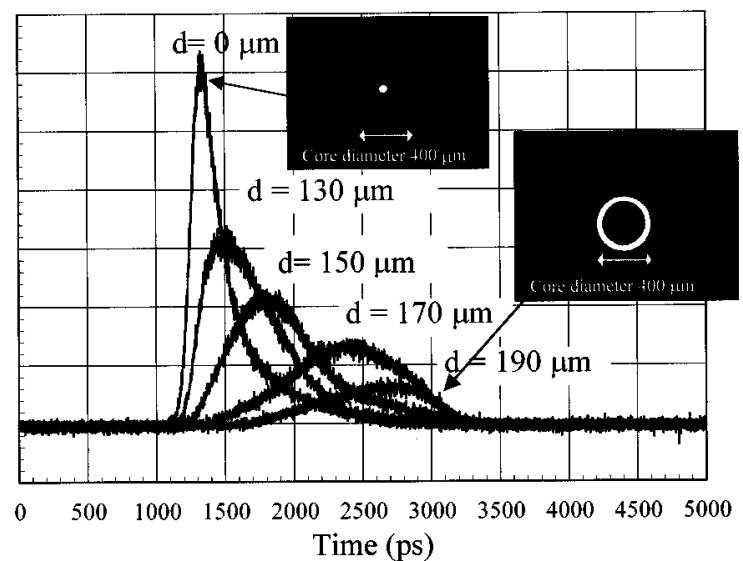

Fig. 5. Measured result of differential mode delay in 100-m PMMA base GI POF. Core Diameter: $400 \mu \mathrm{m}$. Photograph: Output NFP from the GI POF in the DMD Measurement.

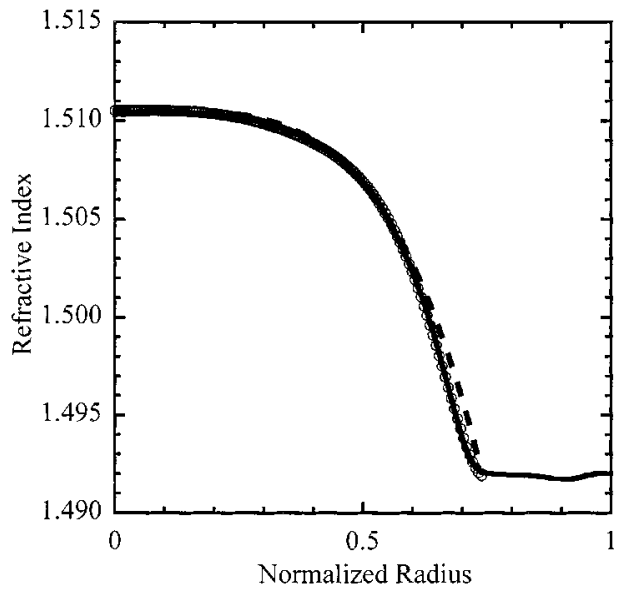

Fig. 6. Measured and approximated refractive index profiles of the PMMA base GI POF used in DMD measurement. Solid line: measured refractive index profile. Broken line: Approximated profile by power-low form when index exponent $g=4.5 . \circ$ : Approximated profile by polynomial form.

of GI POF used is shown in Fig. 6. In Fig. 6, the approximated index profile by the power-law equation shown by (1) is also indicated. Approximated index exponent $g$ of the index profile shown in Fig. 6 is 4.5, which is a little larger than the optimum value, 2.45. The picture of output near-field patterns (NFP's), which directly relate to the mode number of transmitted mode, are also shown in Fig. 5. When the higher order modes are selectively launched by shifting the launch position $190 \mu \mathrm{m}$ apart

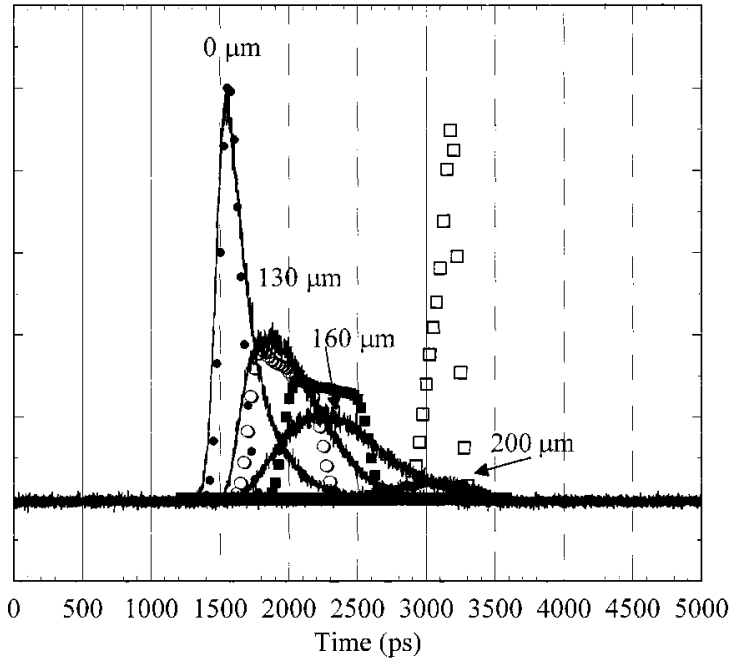

Fig. 7. Comparison of measured and calculated differential mode delay in 100-m PMMA base GI POF. Solid line: measured DMD. •: Predicted DMD (launched at the point where $0 \mu \mathrm{m}$ shifted from the core center). $\circ$ : Predicted DMD (launched at the point where $130 \mu \mathrm{m}$ shifted from the core center). Predicted DMD (launched at the point where $160 \mu \mathrm{m}$ shifted from the core center). $\square$ : Predicted DMD (launched at the point where $200 \mu \mathrm{m}$ shifted from the core center).

from the core center, the ring pattern, which is typically observed in higher order modes, is clearly observed.

It was reported that the relationship between the launch position and the mode number of the multimode optical fiber is described as [7], [8]

$$
\frac{m}{M}=\left[\left(\frac{r}{a}\right)^{g}+\left(\frac{\sin \theta}{\sin \theta_{c}}\right)^{2}\right]^{(g+2) / 2 g}
$$

where $m$ is defined as principal mode number $(m=2 \mu+\nu, \mu$ is the radial mode number), $M$ is the maximum principal mode number, " $a$ " is core radius, $\theta$ the launch angle with respect to the normal to the fiber end, and $\theta_{c}$ is the maximum launch angle of the propagating mode. Since the launch angle $\theta$ is regarded as zero in the launching by the single mode fiber in the DMD measurement, (10) can be simplified as

$$
\frac{m}{M}=\left(\frac{r}{a}\right)^{(g+2) / 2} .
$$

Now, using the relationship shown in (11), the principal mode number of excited modes by the single mode fiber at position $r$ from the core center can be calculated. The group delay of each mode in the GI POF shown in Fig. 5 is predicted theoretically by WKB numerical computation method as described above. In the calculating process, no mode coupling was taken into account. Calculated group delay is shown in Fig. 7 compared with the measured results. It should be noteworthy that the experimental delay time of each mode group is precisely consistent with the theory. These results indicated that the little energy transfer between high and low order modes exists and each mode independently transmit through the GI POF, while the power of high order mode significantly decreases. Therefore, it was confirmed that the mode coupling effect is very small in the PMMA base GI POF, whereas the differential mode attenuation effect should be taken into consideration. 


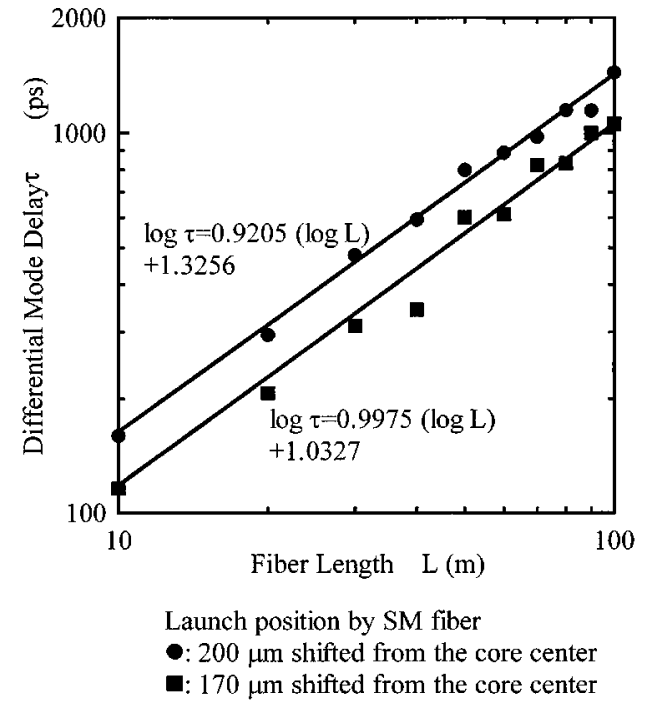

Fig. 8. Time delay of the high order mode group compared with the lowest order mode versus Fiber Length of the GI POF (SI POF Launch). : launched at the point where $200 \mu \mathrm{m}$ shifted from the core center. $\mathbf{m}$ : launched at the point where $170 \mu \mathrm{m}$ shifted from the core center.

The time delay of high order modes compared with the lowest order modes was plotted against the fiber length in Fig. 8. In Fig. 8, the time delays of two mode groups launched at the points of $170 \mu \mathrm{m}$ and $200 \mu \mathrm{m}$ apart from the core center, which were very close to cutoff modes were plotted. A linear relation between the time delay and fiber length is observed, and the slope is almost 1, which also indicates weak mode coupling [9].

\section{FFP from the Fiber Numerical Aperture}

As shown in Fig. 7, it was found that each propagating mode in the GI POF was transmitted independently with little energy transfer among the modes. Therefore, the large difference of the NFP between the low order modes and high order modes that are selectively launched is observed. On the other hand, such NFP difference cannot be observed in the case of the SI type fiber, because there is no relation between the NFP and the launching position. Namely, all modes from lowest order to highest are radiated from any points in the end face of SI type fiber. This is theoretically confirmed from (10) by substituting infinity for the index exponent $g$. The output NFP from 1-m length SI POF is shown in Fig. 9, where the SI POF is launched by using the single mode silica fiber. It should be noted that the output optical intensity from 1-m SI POF is uniform in whole core region although only the small mode groups are launched by the single mode fiber.

In the case of the SI optical fiber, radiation angle of mode, which directly corresponds to the far-field pattern (FFP), is dependent on the mode number. With increasing the mode number, the radiation angle of mode increases. Therefore, the transmission distance dependence of the FFP of SI POF was investigated. Here, the collimated beam from the SELFOC lens was coupled into the conventional SI POF parallel to its axis, by which very low numerical aperture launch $(\mathrm{NA}=0.06$ corresponding to the launch angle of approximately $5^{\circ}$.) was achieved, and the FFP profile was measured after 50-, 40-, 20-, and 10-m transmission. Measured profiles after 40- and 10-m transmission are shown in

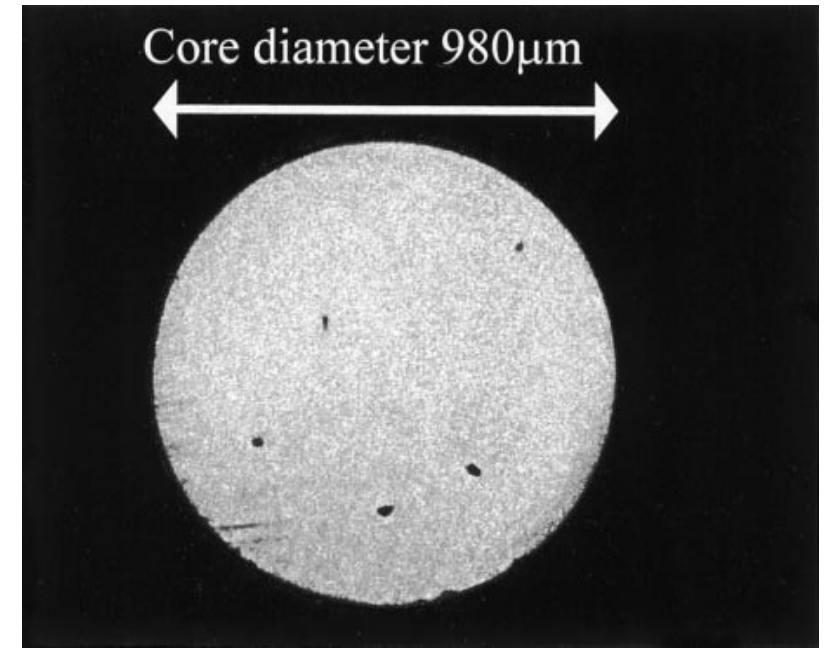

Fig. 9. Near-field output profile from 1-m length SI POF when it is launched by the SM silica fiber.

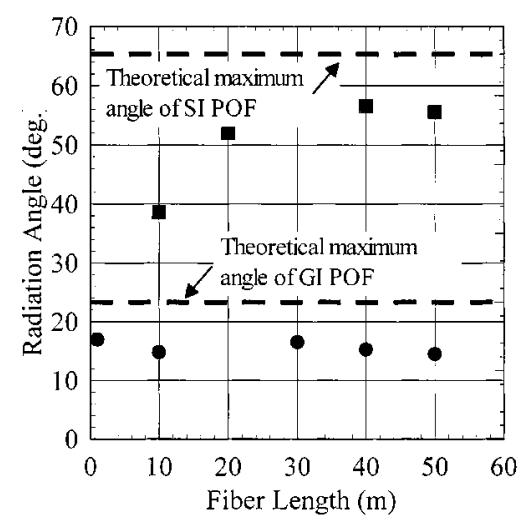

Fig. 10. Fiber length dependence of the radiated angle from conventional SI POF and GI POF when the collimated light (launch angle is around $5^{\circ}$ ) was injected into POF.

Fig. 10. For comparison, the results of 50- and 10-m GI POF are also shown in Fig. 10. As shown in Fig. 10, increase of radiation angle was observed in the SI POF only after 40-m transmission, whereas such broadening of the radiation angle was not observed in the GI POF even after 50-m transmission. The broadening of the output radiating angle observed in the SI POF can be explained by the energy transfer from low order mode to high order mode. On the other hand, it can be said from Fig. 10 that little mode coupling exists in the GI POF. Length dependence of the radiation angle from SI and GI POF's is summarized in Fig. 11. Even in the GI POF, it is supposed that the mode coupling would more strongly affect its bandwidth characteristics if the transmission length is longer than $100 \mathrm{~m}$. However, the transmission distance of the PMMA base POF is limited to less than $100 \mathrm{~m}$ due to its large attenuation. In such a short distance, it can be emphasized that little mode coupling effect on the bandwidth was observed. Regarding the reason why the stronger mode coupling exists in the SI POF rather than GI POF, there have been several hypotheses proposed, for instance the effect of the irregularity of core cladding boundary, phase matching condition between the adjacent modes, etc. [10]. However, these were discussed as the characteristics of silica base 


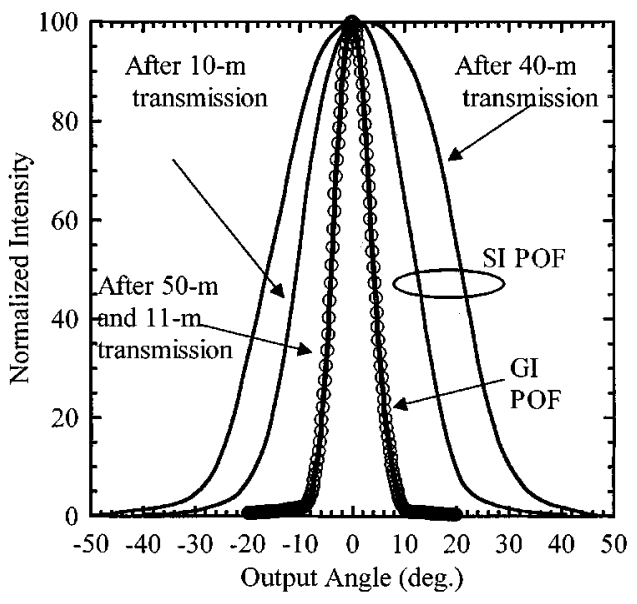

Fig. 11. Comparison of fiber length dependence of the FFP of the conventional SI POF and GI POF. Solid line: after 50-m GI POF transmission. o: after 11-m GI POF transmission.

multimode fiber. Since clarifying the origin of such mode coupling in POF is beyond the scope of this paper, detail of this issue will be presented in our next paper.

\section{E. Differential Mode Attenuation}

Differential mode attenuation was directly measured with using the modified method of the DMD measurement. Similar to the DMD measurement, small mode groups were launched via a SM fiber butted to the one end of GI POF. The attenuation distribution with respect to the fiber $(\mathrm{m} / \mathrm{M})$ in $(10)$ was measured. Since the numerical aperture (NA) of the GI POF varies in the radial direction according to the refractive index profile, acceptable light power, which is highest at the core center, gradually decreases from the core center to periphery. In order to compensate such launch power difference, cut-back method was adopted for differential mode attenuation measurement. Measured result of the differential mode attenuation of the GI POF whose index profile is shown in Fig. 6 is shown in Fig. 12. The attenuation of the low order modes is about $150 \mathrm{~dB} / \mathrm{km}$, while gradual increase was observed with increasing the mode number. It is indicated from Fig. 12 that approximately 17 $\mathrm{dB}$ of power difference exists between the lowest and the highest order mode after 100-m transmission. By considering the differential mode attenuation as shown in Fig. 12, output pulse waveform from 100-m GI POF was calculated with using WKB computation method as described above. The result is shown in Fig. 13. Excellent agreement between the measured and calculated waveforms is observed. Furthermore, the output pulsewidth $\sigma$ was theoretically calculated by WKB numerical computation method in which only the effect of directly measured differential mode attenuation was taken into account. Calculated results when the fiber lengths are 10, 20, $40,50,60,65,70,75$, and $80 \mathrm{~m}$ are shown by open circles. It should be noted that the slope of the relation between the fiber length $L$ and the calculated output pulse width becomes 0.8 as shown in Fig. 14. It should be emphasized that the effect of the differential mode attenuation seriously decreases the exponent value $\gamma$ of the relation between $\sigma$ and $L^{\gamma}$.

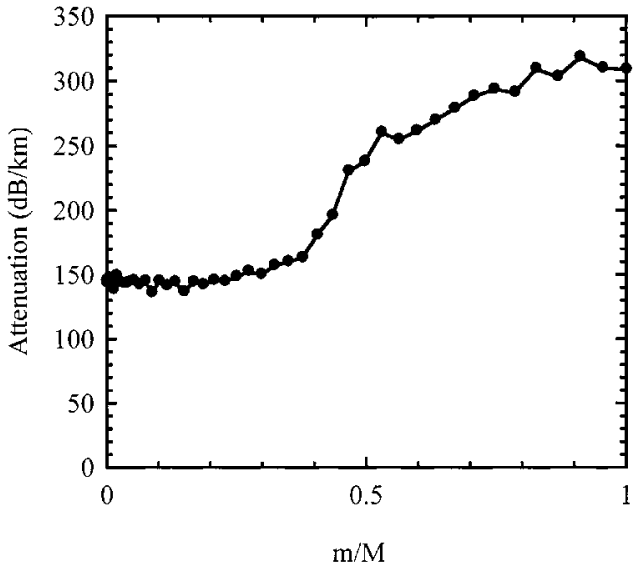

Fig. 12. Measured differential mode attenuation of the PMMA-based GI POF.

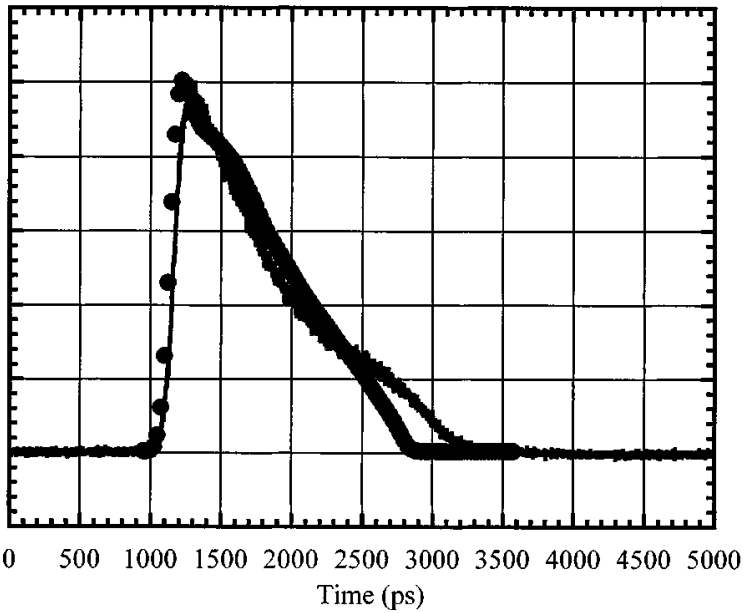

Fig. 13. Comparison of measured and calculated bandwidth characteristics of the GI POF whose index profile is shown in Fig. 6. Solid line: measured waveform. : Predicted waveform taking into account the differential mode attenuation shown in Fig. 12 when index profile is approximated by polynomial form.

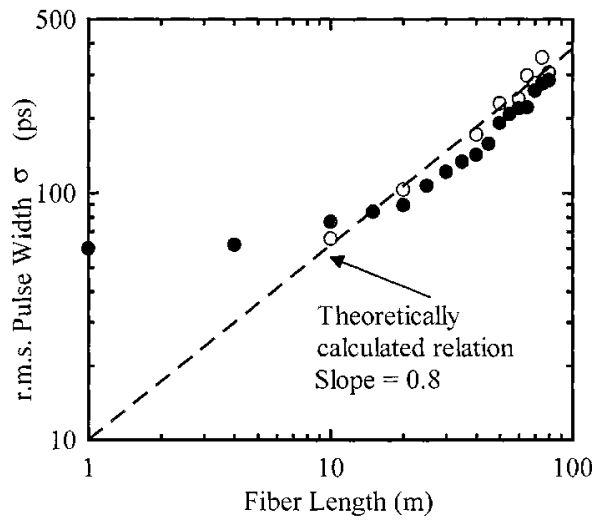

Fig. 14. Calculated relation between the output pulse width and fiber length of the GI POF. : Measured output pulsewidth. o: Calculated output pulsewidth.

\section{CONCLUSION}

It was theoretically and experimentally confirmed that the bandwidth characteristics of the PMMA-based GI POF is strongly influenced by the differential mode attenuation and 
not by the mode coupling. The conclusion that the effect of differential mode attenuation is much large rather than the mode coupling on the bandwidth characteristic of the PMMA base GI POF is derived from the following three reasons:

1) each modes are transmitted independently in the DMD analysis even after 100-m transmission, and their delay times are precisely predicted by the group delay calculation in which no mode coupling effect were considered;

2) linear relation between the time delay of high order modes compared with the lowest order mode and fiber length $L$ was observed in the DMD analysis;

3) output pulse waveform theoretically estimated by considering the differential mode attenuation effect showed an excellent agreement with the experimentally obtained output pulse waveform.

Since the PMMA-based POF link length is considered to be less than $100 \mathrm{~m}$ because of its attenuation property, the result of the weak mode coupling effect indicates that the steady state mode distribution is not necessarily established in the PMMA base GI POF. Therefore, these propagating mode characteristics are very important issues for designing the POF data link system.

\section{REFERENCES}

[1] Y. Koike, T. Ishigure, and E. Nihei, "High-bandwidth graded-index polymer optical fiber," J. Lightwave Technol., vol. 13, pp. 1475-1489, July 1995.

[2] T. Ishigure, M. Sato, O. Takanashi, E. Nihei, T. Nyu, S. Yamazaki, and Y. Koike, "Formation of the refractive index profile in the graded index polymer optical fiber for gigabit data transmission," J. Lightwave Technol., vol. 15, pp. 2095-2100, Nov. 1997.

[3] D. Gloge and E. A. J. Marcatili, "Multimode theory of graded-core fibers," Bell Syst. Tech. J., vol. 52, pp. 1563-1577, 1973.

[4] D. Marcuse, "Calculation of bandwidth from index profiles and optical fibers. 1: Theory," Appl. Opt., vol. 18, pp. 2073-2080, 1979.

[5] — , "Coupled mode theory of round optical fiber," Bell Syst. Tech. J., vol. 52, no. 6, p. 817, 1973.

[6] D. Gloge, "Impulse response of clad optical multimode fibers," Bell Sys. Tech. J., vol. 52, no. 6, pp. 801-816, 1973 .

[7] R. Olshansly and S. M. Oaks, "Differential mode attenuation measurements in graded-index fibers," Appl. Opt., vol. 17, no. 11, pp. $1830-1835,1978$

[8] D. Marcuse, Principle of Optical Fiber Measurements. New York: Wiley,, 1973.
[9] W. R. White, M. Dueser, B. Read, and P. Wiltzius, "New measurement techniques for POF material and performance," in Proc. 7th Int. POF Conf.' 98, Berlin, Germany, Oct. 1998.

[10] H. Kajioka, "The theoretical and experimental study of mode-coupled graded index fibers based on scattering matrix method," Trans. IECE Japan, vol. E64, no. 3, pp. 203-209, 1981.

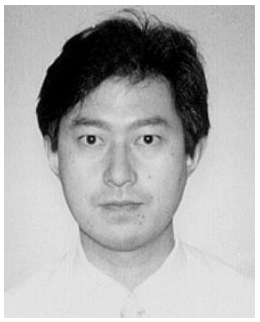

Takaaki Ishigure was born in Gifu, Japan, on July 30, 1968. He received the B.S. degree in applied chemistry and the M. S. and Ph.D degrees in material science from Keio University, Japan, in 1991, 1993, and 1996 respectively.

$\mathrm{He}$ is currently an Instructor of Keio University, Japan. His current research interest is in fabrication and system application of the graded-index polymer optical fiber.

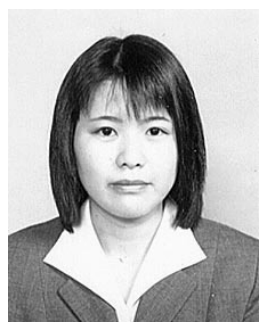

Mariko Kano was born in Hiroshima, Japan, on October 19, 1974. She received the B.S. degree in applied chemistry and the M. S. degree in material science from Keio University, Japan, in 1998 and 2000, respectively.

She is currently an Engineer of Sony Corporation, Japan. Her current research interest is in the system application and network of the polymer optical fiber.

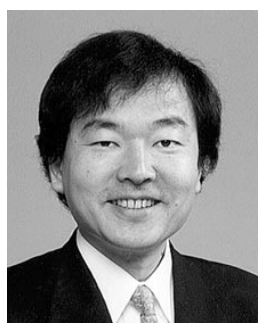

Yasuhiro Koike was born in Nagano, Japan, on April 7, 1954. He received the B.S., M.S., and Ph.D. degrees in applied chemistry from Keio University, Japan, in 1977, 1979, and 1982, respectively.

He has been a Professor of Keio University and developed the high-bandwidth GI polymer optical fiber, he has concurrently been the leader of Plastic Optical Fiber project of Telecommunications Advancement Organization (TAO) of Japan since 1998. He stayed as a Visiting Researcher at AT\&T Bell Laboratories from 1989 to 1990 .

Dr. Koike received the International Engineering and Technology Award of the Society of Plastics Engineers in 1994. 